\title{
The Meanings of Materials: The Case of Bowl
}

\author{
Oğuzhan Güngör
}

\begin{abstract}
Products which are used in daily life can be attributed different meanings by users. There are some studies that approach this meaning difference from different points of view. While Jean Baudrillard tries to explain this meaning difference through the values attributed to products, Krippendorf states that products can be attributed different meanings in different contexts and that people make sense of the products through those contexts. The attributed meaning on the product does not proceed only from the function of that product. There are different factors affecting the meanings and meanings can change according to those factors. While attributed meanings can be related to some factors such as the form, color, smell and texture -which are related to the product itself, some sociological and psychological factors such as culture, the past and the personality characteristics of the user may also lead to those different attributed meanings. In this study, the meanings of five different materials -which enable a product to become concrete- are studied in the eyes of the user, with the technique of semantic differentiation. The materials are determined as metal, ceramic, polymer, glass and wood.
\end{abstract}

Keywords - product semantic, material, meaning, making sense

\section{INTRODUCTION}

Klaus Krippendorff studies on what design is and he says "design is making sense of things." [1]. Product semantics is a study which investigates the symbolic qualities of the usage of artefact forms in a cognitive and social context and the implementation of this acquired information on industrial product objects. Krippendorff emphasizes that product semantics is not a style, a program or a movement. Instead, he emphasizes that product semantics deals with the meanings of the artefacts on users [1]. Krippendorff analyses sense-making in four contexts: Operational context, sociolinguistic context, context of formation and ecological context.

\section{MAKING SENSE OF THE OBJeCtS}

In the operational context, people are seen in an interaction while using the object. In the operational theory of meaning, it has to be explained how the forms of the objects create a perception over the user within the frame of their uses. For this reason, identities are to be investigated through various semantical aspects such as qualities, tendencies, simplicities, logic and motivations [1].

In the sociolinguistic context, people are in communication

Manuscript received Nov. 20, 2019.

O. G. Author is with the Department of Industrial Product Design Istanbul Technical University (ITU) Faculty of Architecture, Taskisla Taksim, Istanbul 34437, Turkey. with one another about particular objects. This communication is about the usage of objects and their relation with users. In this way, the components of the objects which help configuring (which means the qualities of users who use the object to make sense of it, defined with the cognitive information of them) become the principal parts to make sense of that object. The operational and sociolinguistic contexts are related to the form of the object and its function [1].

In the context of formation; designers, producers, distributors, users and other relevant subjects are considered as common participants who, in different ways, contribute to the technical organizations of cultural and material entropy, in creating and consuming objects [1].

In the ecological context, objects are seen in an interaction with their populations, which contributes to the self-production of technology and culture [1]. Krippendorff establishes a metaphorical relationship with biological ecosystem, for us to understand human-made objects better. Biological ecosystem is established upon the interaction of a living being with itself and with another living being. However, people are at the top position in the ecosystem of human-made objects. Objects are to serve people while interacting with themselves and other objects in the meantime. Humans are the uppermost directors of this ecosystem. Human-made objects can change so fast, so do their meanings. An object serving a specific function, in time, can even become used for another function and this occurs through the meaning attributed to the object [2].

This change of the meaning is also approached by Baudrillard, with another point of view.

Baudrillard mentions the values and meanings attributed to objects in his book "The System of Objects". An object can have a functional value, an economical change value, a sign value and a symbolic value [3]. However, this study is based on Krippendorff's approach. According to Krippendorff, this ecosystem can be viewed in two main topics: Diachronic and synchronic.

From the diachronic perspective, objects appear to carry the traces of their own evolutions. The sense-making is made with the historical process of that object. An object may change over time and it can fulfill the requirements of the conditions of that time. The object that has been changed is not considered as a new type in the diachronic perspective. It can be thought as an evolved version of the object's previous situation [2]. In order to explain this better, Krippendorf gives the example of telephone. From the invention of telephone until today, its function has remained the same but also it has developed and led to changes with the help of technology. When telephone was first launched, it reached a universal form, then in time it has 
become varied in itself but the first form of it was referenced in a way. With the developing technology, firstly it has become wireless and now it is mobile. The dominant form of telephone has completely changed. If one does not view this transition process from the diachronic perspective, it is hard to establish a relationship between the first telephone and the telephone we use at present. The diachronic approach enables us to solve this connection [2]. Similarly, one can also approach camera diachronically. Although new digital cameras do not make any mechanical sound during shooting, they are digitally provided a sound in order to give a reference to the origin of camera.

As for the synchronic perspective, an object also has sub-systems. For instance, communication technologies exist with many sub-systems. The relationship between these systems and communication technologies and also their collaborative work constitute a particular ecosystem. In the perspective of synchronic ecosystem, there are four main connections: Casual connections (as in the example of telephone, physical connections such as cable and satellite which are required for telephone to work), family resemblances (dining table, center table and nesting table can be shown as examples to that), metaphorical connections (the metaphorical use in e-book can be an example to that, as it is an electronical reading device which is metaphorically associated with book) and lastly institutional liaisons (that is an object's need for other institutions in order to maintain its existence;like television, which does not function without satellite, broadcaster, camera, etc. and it gathers different institutions) [2].

In this study, meaning differences of materials are examined. Users are expected to evaluate metal, ceramic, glass, polymer and wooden, on the basis of Krippendorff's contexts and these are operational context, sociolinguistic context and ecological context. Ecological context is dealt with synchronic point of view and the object taken as an example is a bowl. Also with the synchronic perspective, the difference in materials not only constitutes the sub-system of the object but also this product made of different materials constitutes a family of products in some way. Here, the context of formation is not taken into consideration. If the sense-making is made according to operational context, the technological histories of materials and their productions must be taken into consideration. This study is based on the sense-making of crude materials. Therefore, the operational context is excluded from the sense-making.

\section{MAKING SENSE OF THE MATERIALS}

It was explained above how an object is made sense of, according to Krippendorff. In brief, one makes sense of an object and categorizes it through different contexts. What allow this are the signs that a product carries when we first see it. These signs allow us to define the object and categorize it in a way. While doing this, we act through the contexts mentioned above. Products carry these signs in order to deliver a message to users. The designer sends these messages to the receiver, who is a user, via the product. The meaning of a product is constituted depending on its form, function, color and the relationship between its all other features. How these messages are interpreted can vary from person to person, society and culture [4].

Product designers are in a search of product personality and they make a selection of materials using various methods. Product personality and identity also take place in operational context. Choi evaluates material selection according to their meanings from five different angles. These are emotional, relational, perceptual, sensory, physical and usage [5]. While making sense of products, in what context the objects are dealt with is important. Krippendorff uses the example of chair in order make this clear. When a chair is always used in an office and its relationship with that environment is reinforced, the chair now gains the meaning of being an office chair. The chair's features such as its form and material now represent an office chair. Krippendorff thinks that the same chair's being used in a family house in daily life will be at a variance with the previously formed office context. While making sense of objects, we tend to do it in a cognitive way. Our former knowledge and experiences, the society and the environment we live in can be presented as the factors which influence our sense-making process [1].

Material is one of the important elements about the product in terms of delivering message and sense-making. Material constitutes a key element in product design. Choosing the material that will be used as the product is designed, becomes crucial in terms of both the technical features of the product and the communication between the product and the user. Van Kesteren evaluates this relationship as an interface. He emphasizes that the material which takes on the task of being an interface between the user and the product must be chosen correctly and effectually, in order to enhance the usability [6]. Users sense the materials used in products with sense organs. The material we sense with sense organs shows a stimulus effect on our feelings. Additionally, the user's sensorial relationship with the material influences the user's evaluation of quality on the product to a considerable extent. As it was mentioned above, this sensorial relationship occurs via our sense organs; through sight, touch, taste, hearing and smell [6].

Elvin Karana and Paul Hekkert state that these sensorial stimulations of the material are due to its technical characteristics. The ability of a material to take a smooth, bright, firm, soft shape and to take the forms of different geometrical figures, gives a reference to the character of the material. The sense-making is not made only with this sensorial stimulation; culture also has a considerable effect on this. According to a research from which Elvin Karana and Paul Hekkert cite, people who live in Turkish culture generally identify a chest made of wooden with a dowry chest. In this case, since the usage of wooden in the manufacturing of a chest is typically identified with another product used widely in that particular culture, it is seen that the meaning towards the product changes when the wooden material is used with another products coming from the same family [7]. In this context, sociological factors like culture are also the differential features in the sense-making of a 
material. In this study, cultural difference is not mentioned.

The color, texture and weight of the product and the sounds that it makes, contribute to the usability of the product and to the experience of usage. Product designers may prefer different materials in order to create these sensory perceptions [8]. They can even provide some functions directly through the material. Besides, the product's remaining in the same form can be provided with the material that will be used [4]. The selection of material affects the way users interact with it. For instance, when materials having high thermal conductivity are used in a tea glass, it is difficult to hold it with hand. For this reason, the user can change the practice of using the product or the designers can try to prevent this heat transmission by changing the form [6]. The tea glass known as slim waisted and its handle added version can be examples of that.

A thing needs a form and this form should make a sense for the thing to be apprehensible and useable. Sense-making always requires the sense-maker (a person) to refer to his/her cognitive process. Then, the function of a thing is related to the meaning of the thing. In this way, one can say "Form follows meaning." instead of 'Form follows function." Because the function of the thing may gain a meaning in a different way regardless of the function which the designer suggests and it can have a function through the meaning it has gained [1]. If we are to think about this sense-making in terms of material, instead of function, one can say "'Material follows meaning. "' just as 'Form follows meaning. "' In this context, material has a very important role in product designing. If there are problematic lives in the interface which emerge through the relationship between material and product, they cause a lack of communication with the user. This situation causes problems in the usability of that product. Another factor affecting this communication is the compatibility between the product and its material, in the context of product identity and personality[6]. Ashby and Johnson make discussions about the personality of materials. They make an assumption on that a material's personality occurs only when the material is used on a product. They compare the material to an actor and emphasize that materials can disguise different personalities according to the product. They explain this situation through the example of glass. While the glass used in a camera lens is associated with engineering, the glass used in a beer bottle is just an expendable material. Similarly, while golden mostly represents power and wealth, when it is used in micro circuits, it stands out with its technicality [9]. Ashby and Johnson make inferences about the meanings of metal, ceramic, polymer, glass and wooden [9].

Metal material is defined as cold, clear and flawless by Ashby and Johnson. It has a reflective characteristic when its surface is polished. It is qualified as a reliable and powerful material since it is widely used in different fields of industry. It can be shaped in many

ways thanks to its material characteristic, which popularizes the use of metal material [9].

Ceramic and glass are considered as traditional materials. Greek pots and Roman glasses reinforce this traditional idea.
Their ability to be colored and be durable against the dangers of outer environment almost give them immortality. Apart from their being traditional materials; glasses and ceramics are widely used in high-tech products [9].

As for polymer materials, they are qualified as '"cheap plastic imitations '. Polymer materials, given color and texture, have the ability to imitate different materials. However, they are not always able to demostrate in themselves the strengths of the material they imitate. According to Ashby and Johnson, polymer materials remain warmer, compared to metal and glass materials [9].

Wooden is a natural material and it has its own texture, pattern, smell, sound and color, which are peculiar to itself. These create an integrity of senses and feelings. According to Ashby and Johnson, wooden material is warmer and softer compared to many other materials. Wooden is also associated with craft at the same time [9].

As it is mentioned in Ashby and Johnson's works; due to the productions, usages and characteristics of different materials, they have different identities and meanings. In this context, material selection and the relationship between material and product play an important role. This study aims to contribute to material selection in product designing, by discussing in what ways materials carry meanings.

\section{Methodology And Method}

The methodology and theoretical substructure of this study are based on Krippendorff's semantic approach to human-made objects. As it is stated also in the part of literature knowledge, objects gain meanings according to contexts. These contexts are specified as operational, sociolinguistic, formation and ecological. The contexts which this study is located are operational, sociolinguistic and ecological. In the ecological context, synchronic point of view is dealt with. Besides, also theoretical studies based on the meanings of materials, show that materials used in products have different meanings. In this study, meaning differences between bowls made of five different materials are tried to be found out.

The technique of semantic differentiation is used in order to evaluate the meaning difference on people. Bowls made of five different materials are evaluated by participants, by means of 12 adjectives consisting of binary oppositions. These 12 binary oppositions are presented in Table 1.

35 students ranging in age from 18 to 25 participated in the study. However, two of those participants indicated that the material did not have any sex, so they did not evaluate the material in feminine and masculine terms. These participants were eliminated in order not to affect the general result and the analysis was carried out with 33 participants. All students were freshmen studying at the department of Industrial Design at Istanbul Technical University. Participants had taken the "Material in Design" lecture which was compulsory. Application was made in the final class of the term and in the classroom where the class had been taught. This means the students were supposed to know material knowledge. The 
students were observed sitting in the classroom dispersedly. During the study, the application was recorded and photographed, with the permission of the students. Also, some notes were taken at the same time.

Bowls made of metal, ceramic, polymer, glass and wooden were put on a table. Some appetizers and dried fruits were put in these bowls. Then the students were invited to the table in particular groups. The groups were constituted of 6 students, in order to make the sharing of the bowls between the students easier. The scenario below was told each of the groups.

'Imagine yourselves as if you were at a cocktail party given for the period-end celebration. There is a table in front of you and on this table there are bowls. During the cocktail party, you all eat from the bowls filled with appetizers and dried fruits, while chatting and communicating with each other. Since the number of the bowls are less than the number of people, you have to share these bowls among yourselves. If you have a bowl in your hand, you hand it to your friend who has no bowl in hand. In this way, you share and interact with each other. At the same time, you both chat and eat from the appetizers and dried fruits. Please do not forget to recognize, sense and touch the bowls while sharing. "'

The primary aim of telling this scenario is enabling the participants to make sense of the object in operational, sociolinguistic and ecological contexts. Participants talked to each other, told their opinions and thoughts about the bowls and the material, during the application. Also, they commented on in which settings and conditions these bowls made of different materials can be used. Due to technical incompetence and deficient planning, comments of the participants could not be recorded. Therefore, those information cannot be interpreted although they are very valuable in terms of sociolinguistic and operational contexts. However, while evaluating the materials, the participants are thought to have considered the conversations between themselves.

After all participants made sure that they experienced the bowls made of five different materials, in order to apply the differentiation technique, they were given a participation form and an evaluation table consisting of 12 binary oppositions. Students were told in what way the evaluation would be carried out and the things they were expected to be careful about. The emphasized point was the evaluation of materials. The participants were told not to evaluate the differences like color and form. The evaluation was graded from 1 up to 7 . Table 1 is the evaluation table. In figure 1 , the bowls made of different materials are seen.

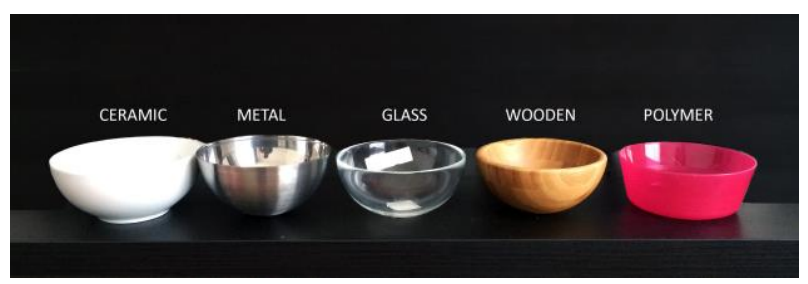

Fig. 1. The bowls made of different materials

\section{ANALYSIS}

The data acquired through the participants was analyzed via SPSS. First, the average value of each material was found out according to related adjectives. Table 1 shows the values. Then, General Linear Model - Repeated Measures was applied. Thus, it was understood among which materials the related adjectives have significant differences. The graphics were created in order to interpret these significant differences better. The sense-making of the participants can be interpreted according to the average values in this way:

Metal material: Considering the average values, metal material is interpreted as aggressive, highly hard, highly cold, highly long-lasting, relatively sexy, masculine, highly serious, irritating and futuristic. Since the results are so close to likert scale $(4,00)$, the sense-making of metal is cheap/expensive, classic/trendy, mature/youthful neutral.

Ceramic material: Considering the average values, ceramic material is interpreted as relatively passive, relatively expensive, relatively cold, relatively long-lasting, feminine, relatively serious, relatively lovable, mature and nostalgic. Since the results are so close to likert scale $(4,00)$, the sense-making of ceramic material is classic/trendy, dull/sexy neutral.

Polymer material: Considering the average values, polymer material is interpreted as passive, highly cheap, classic, highly soft, relatively warm, dull, feminine, highly humorous, highly irritating, highly youthful and relatively futuristic. Since the results are so close to likert scale $(4,00)$, the sense-making of ceramic is disposable/long-lasting neutral.

Glass material: It is interpreted as passive, classic, hard, relatively cold, relatively long-lasting, relatively sexy, serious, lovable and mature. Since the results are so close to likert scale $(4,00)$, the sense-making of glass material is cheap/expensive, feminine/masculine and nostalgic/futuristic neutral.

Wooden material: Considering the average values, wooden material is interpreted as passive, relatively expensive, relatively soft, highly warm, long-lasting, sexy, relatively humorous, highly lovable, mature and nostalgic. Since the results are so close to likert scale $(4,00)$, the sense-making of wooden is classic/trendy and feminine/masculine neutral.

It is analyzed whether there are significant differences among materials against each other according to the adjectives. The evaluation results for each adjective group are presented in the table.

As it is seen in Figure 2, the aggressive/passive evaluation of metal material is different from the other materials at a significant degree. According to this analysis, metal material is aggressive in a significant way, compared to the other materials. 
TABLE I: THE AVERAGE VALUES OF MATERIALS IN BINARY OPPOSITIONS, ACCORDING TO ACQUIRED RESULTS.

\begin{tabular}{cccccc}
\hline \hline $1 / 7$ & Metal & $\begin{array}{c}\text { Cerami } \\
\mathrm{c}\end{array}$ & Polymer & Glass & Wooden \\
\hline Aggressive/Passive & 2,15 & 4,45 & 5,48 & 3,97 & 5,30 \\
Cheap/Expensive & 4,33 & 4,70 & 1,21 & 4,18 & 4,55 \\
Classic/Trendy & 4,21 & 3,82 & 3,64 & 3,15 & 3,94 \\
Hard/Soft & 1,79 & 3,03 & 5,91 & 2,76 & 4,30 \\
Warm/Cold & 5,82 & 4,39 & 3,64 & 4,52 & 1,85 \\
Disposable/Long-lasting & 5,85 & 4,42 & 4,00 & 4,76 & 4,97 \\
Dull/Sexy & 4,61 & 3,94 & 2,27 & 4,61 & 4,91 \\
Feminine/Masculine & 5,33 & 2,97 & 3,42 & 3,79 & 4,27 \\
Humorous/Serious & 5,76 & 4,52 & 2,09 & 4,67 & 3,67 \\
Irritating/Lovable & 3,27 & 4,42 & 2,09 & 4,82 & 5,88 \\
Mature/Youthful & 4,06 & 3,15 & 5,64 & 3,33 & 3,48 \\
Nostalgic/Futuristic & 5,55 & 2,82 & 4,61 & 3,88 & 2,79 \\
\hline
\end{tabular}

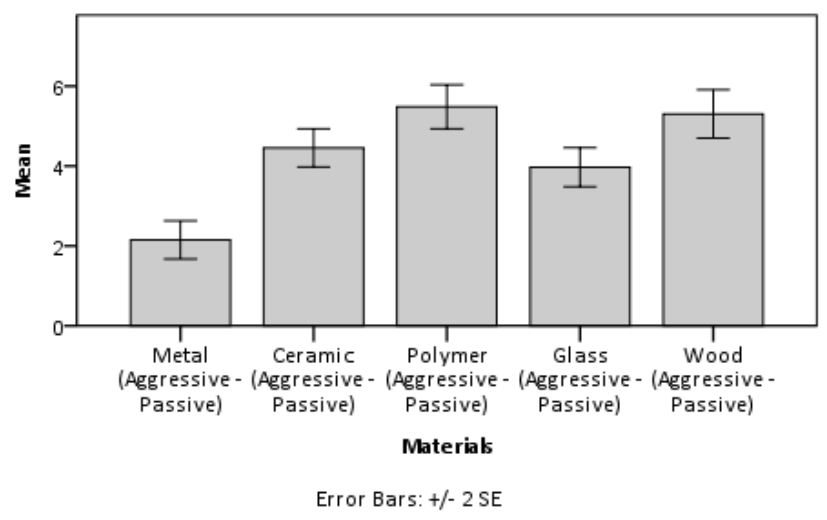

Fig. 2. Aggressive-Passive evaluation

As it is seen in Figure 3, the cheap/expensive evaluation of polymer material is different from the other materials at a significant degree. According to this analysis, polymer material is cheaper in a significant way, compared to the other materials.

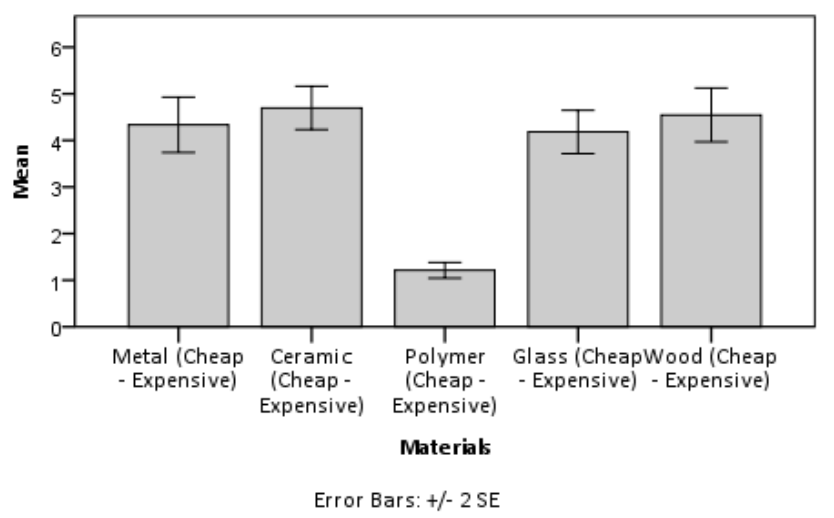

Fig. 3. Cheap Expensive evaluation

As it is seen in Figure 4, there is not any significant difference in the classic/trendy evaluation of materials.

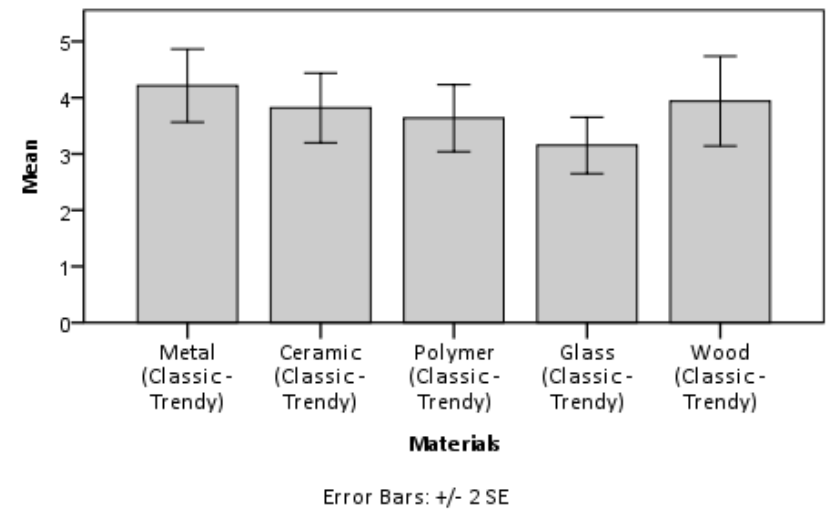

Fig. 4. Classic-Trendy evaluation

As it is seen in Figure 5, the hard/soft evaluation of metal, polymer and wooden material is different from the rest of the materials at a significant degree. Especially metal material obviously appears to be harder than the other materials. As for the polymer material, it is softer than the other materials at a significant degree. While ceramic and glass material are not different from each other at a significant degree; metal, polymer and wooden material are different from each other at a significant degree in the hard/soft evaluation. Ceramic material appears to be softer than metal material but harder than polymer and wooden material. As for the glass material, it is softer than metal material but harder than polymer and wooden material. While wooden material is softer than metal, ceramic and glass material, it is harder than polymer material.

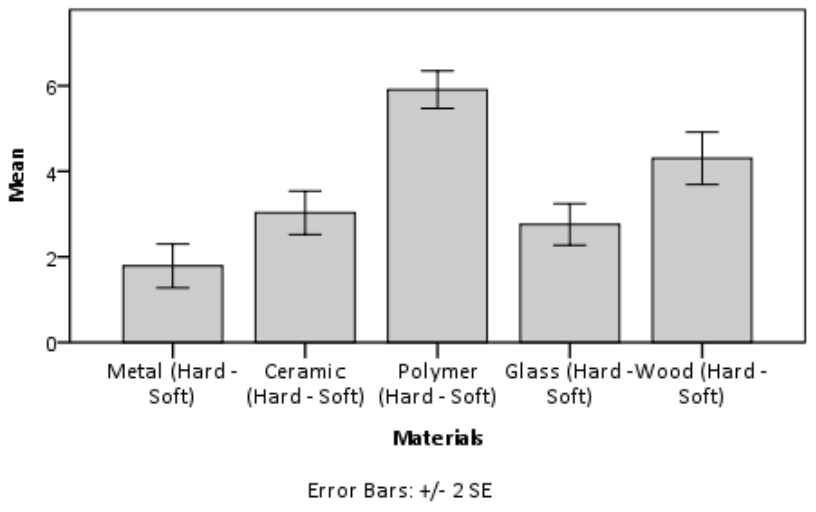

Fig. 5. Hard-Soft evaluation

As it is seen in Figure 6, the warm/cold evaluation of wooden material is different from the other materials at a significant degree. Wooden material is warmer in a significant way, compared to the other materials. 


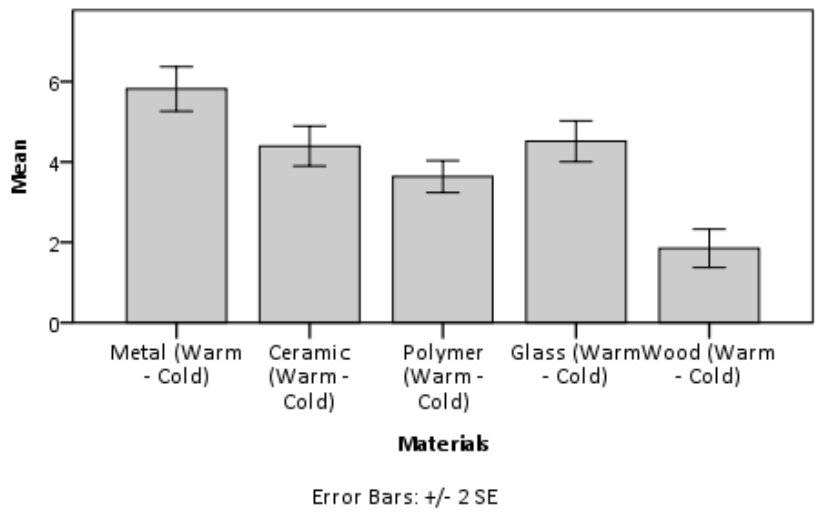

Fig. 6. Warm-Cold evaluation

As it is seen in Figure 7, there is a significant difference in the disposable/long-lasting evaluation of metal, ceramic, polymer, glass and wooden material.

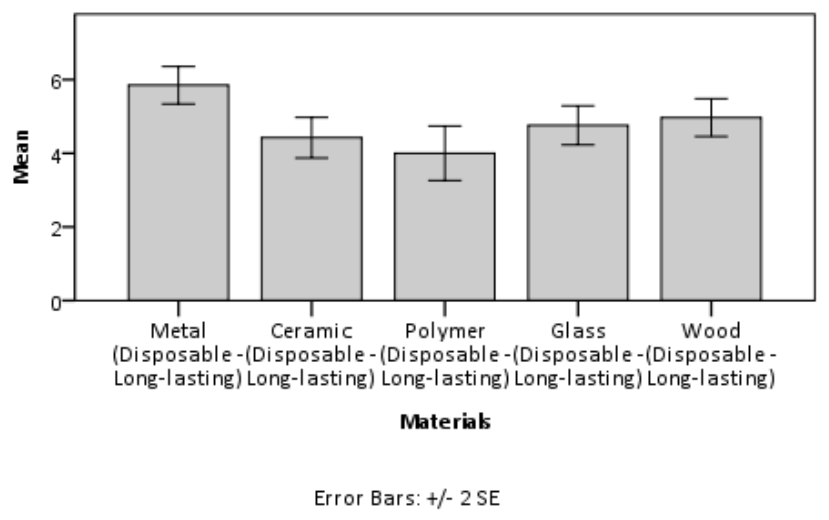

Fig. 7. Disposable-Long-lasting evaluation

As it is seen in Figure 8, polymer material is duller than the other materials at a significant degree.

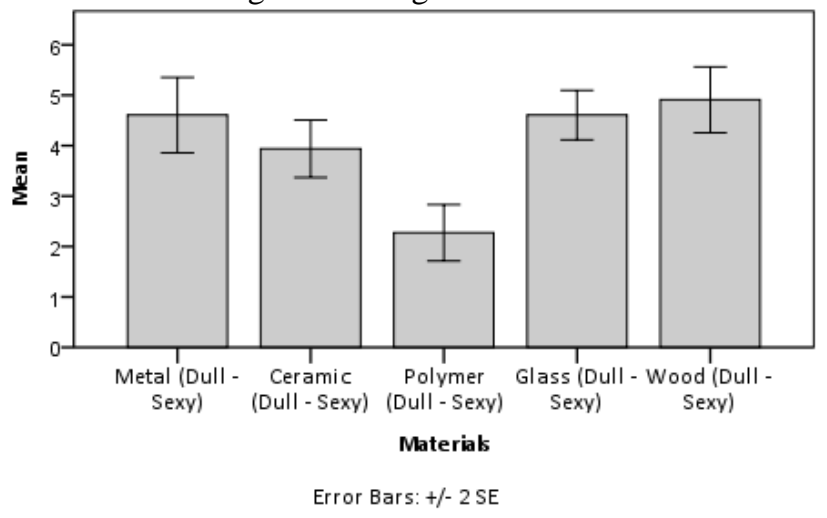

Fig. 8. Dull-Sexy evaluation

As it is seen in Figure 9, the feminine/masculine evaluation of metal material is different from ceramic, polymer and glass material at a significant degree and it appears as masculine. The feminine/masculine evaluation of ceramic is different from metal and wooden material. Ceramic material is considered more feminine compared to metal and wooden material. Wooden material has a significant difference between only ceramic material and itself. Wooden material appears to be more masculine compared to ceramic material.

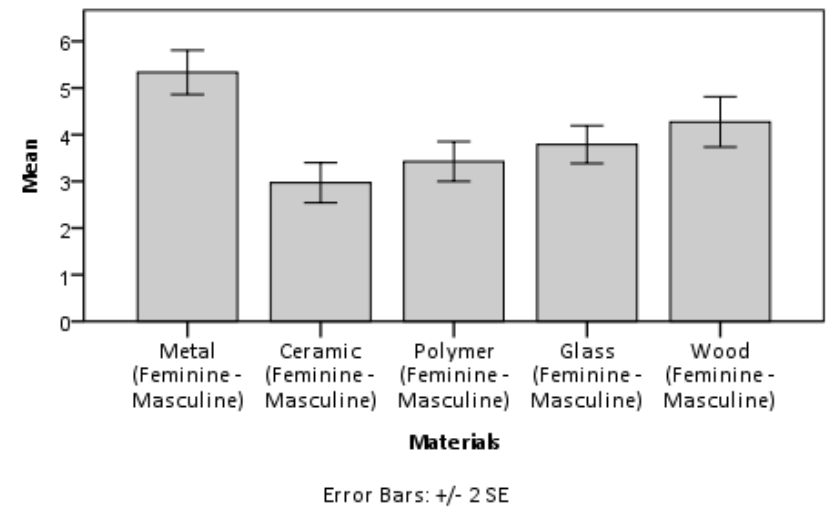

Fig. 9. Feminine-Masculine evaluation

As it is seen in Figure 10, metal material is different from the other materials in the humorous/serious evaluation at a significant degree and it is interpreted as serious. Ceramic material is different from metal and polymer material at a significant degree. Ceramic material is more humorous than metal material but more serious than polymer material. As for the polymer material, it is more humorous than all other materials at a significant degree. Glass material is considered as different from all other materials at a significant degree, except for the ceramic material. Glass material is more humorous than metal material but more serious than wooden material. Wooden material is evaluated as different from all other materials at a significant degree, except for ceramic material. While wooden material appears to be more humorous than metal and glass material, it is more serious than polymer material.

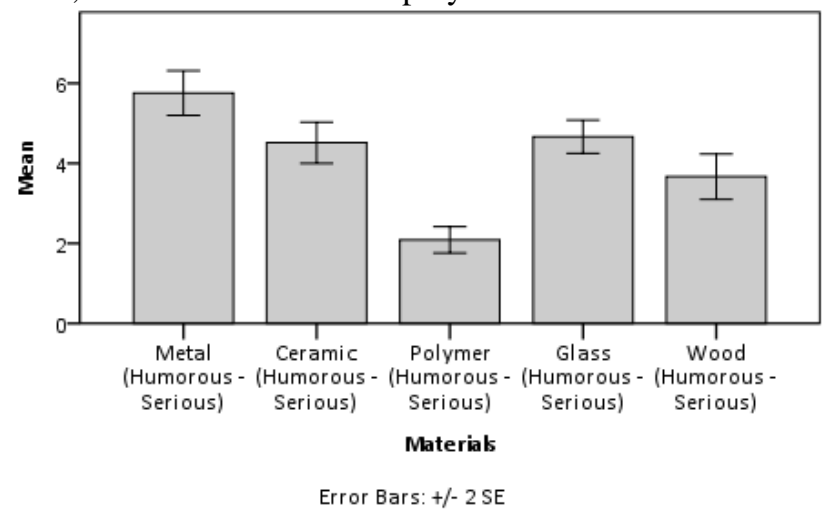

Fig. 10. Humorous-Serious evaluation

As it is seen in Figure 11, metal material is evaluated as different from the other materials except for ceramic material, at a significant degree. While metal material is more lovable than polymer material, it is more irritating than glass and wooden material. Ceramic material is different from polymer and wooden material at a significant degree. While ceramic material is more lovable than metal material, it is more irritating than wooden material. Polymer material is seen lovable compared to all the other materials at a significant degree. As for the glass material, it is different from all the other material at a significant degree, except for ceramic material. While glass material which is generally evaluated as lovable, is more irritating than wooden material, it is more lovable than metal material. As for the 
wooden material, it is evaluated as lovable compared to all the other materials.

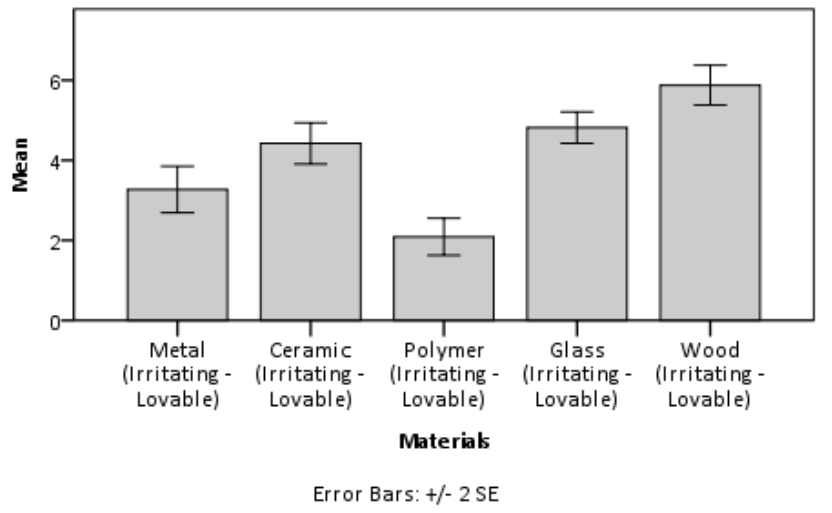

Fig. 11. Irritating-Lovable evaluation

As it is seen in Figure 12, polymer material is different from all the other material at a significant degree and it is evaluated as more youthful.

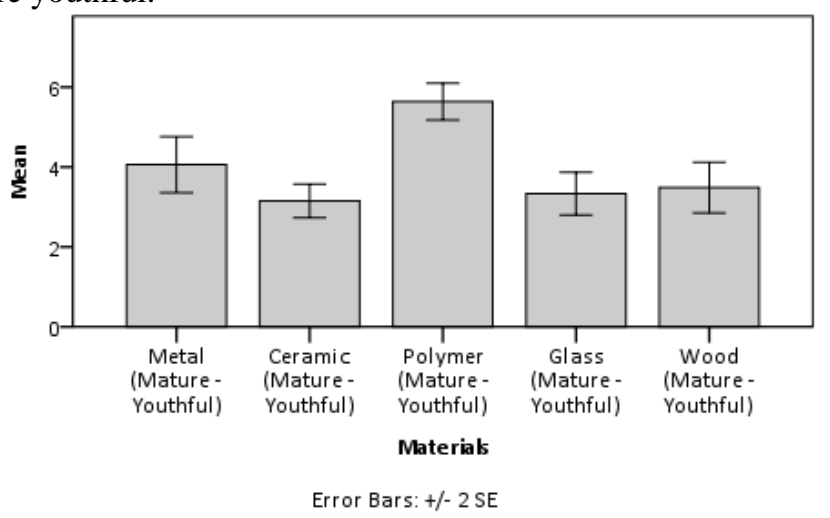

Fig. 12. Mature-Youthful evaluation

As it is seen in Figure 13, metal material is evaluated as more futuristic at a significant degree than all the other materials except for the polymer material. Ceramic material is different from polymer and glass material at a significant degree. Ceramic material is seen more nostalgic than polymer and glass material. Polymer material is different from ceramic and wooden material at a significant degree. Polymer material is considered more futuristic compared to ceramic and wooden material. Glass material is different from only metal material at a significant degree. Glass material is evaluated as more nostalgic than metal material. As for the wooden material, it is different from metal and polymer material at a significant degree. Wooden material is more nostalgic compared to metal and polymer material.

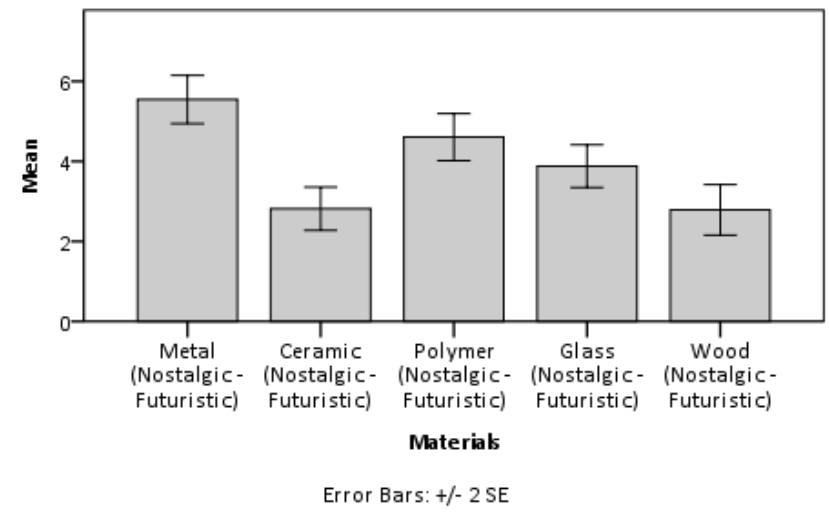

Fig. 13. Nostalgic-Futuristic evaluation

\section{CONCLUSION AND DISCUSSION}

As it can be understood through the results of the analysis, materials are different from the other materials at a significant degree in relation to particular adjectives. These differences have parallels with the evaluations of Ashby and Johnson. It was indicated in the literature part that besides of a product's function and form, its material is also effective in the evaluation of the product. As for this study, it enables us to see that there are differences among the meanings of materials in operational, sociolinguistic and ecological contexts. It is not particularly evaluated in this study that what sorts of meaning differences the material causes on the identity and the personality of product. However, it can be assumed that the meaning differences of products affect the meaning of the product. In the forthcoming studies, the semantic effects of meaning differences on products can be investigated. For the results and the discussions to be productive and for more qualified data; a better construction for the operating procedure and a better planning is recommended.

An instrument, which will make material selection easier, can be developed for the purpose of creating meaning in design, by reporting and visualising the semantic differences of materials in a more systematical way. The acquired knowledge is thought to be able to provide this. Thus, designers can be provided with convenience in material selection within the discipline of industrial design. This semantic differentiation can get more specialised with the enhancement of material diversity. All metal materials or all glass materials are not the same. If we are to give an example, a semantic difference will probably emerge between copper and steel. For this reason, this study can be carried out with more specific types of materials instead of generalizing them as metal, polymer etc.

Cultural differences and the setting where this study was made are also considered as factors which can change the result. Another study can be made in order to research these factors.

The participants were assumed to have material knowledge. It is not known whether the results would be the same in a condition in which the participants do not have any material knowledge. Another subject that can be investigated is the differences between the sense-making of the participants who have material knowledge and the sense-making of the ones who 
do not have any material knowledge.

\section{ACKNOWLEDGMENT}

I would like to express my gratitude to Asst.Prof.Dr. Ozge MERZALI CELIKOGLU who is instructor of the course which is Design Semantics.

\section{REFERENCES}

[1] Krippendorff, K., On the essential contexts of artifacts or on the proposition that" design is making sense (of things)". Design issues, 1989. 5(2): p. 9-39. https://doi.org/10.2307/1511512

[2] Krippendorff, K., The semantic turn: A new foundation for design. 2005: crc Press.

https://doi.org/10.4324/9780203299951

[3] Baudrillard, J., Nesneler Sistemi, çev. Oğuz Adanır, Aslı Karamollaoğlu, 2011. 2.
[4] Karana, E., P. Hekkert, and P. Kandachar, Meanings of materials through sensorial properties and manufacturing processes. Materials \& Design, 2009. 30(7): p. 2778-2784. https://doi.org/10.1016/j.matdes.2008.09.028

[5] Choi, J., Material selection by the evaluation of diffuse interface of material perception and product personality. International Journal on Interactive Design and Manufacturing (IJIDeM), 2017. 11(4): p. 967-977. https://doi.org/10.1007/s12008-016-0320-9

[6] Van Kesteren, I., A USER-CENTRED MATERIALS SELECTION APPROACH FOR PRODUCT DESIGNERS. METU Journal of the Faculty of Architecture, 2010. 27(2). https://doi.org/10.4305/METU.JFA.2010.2.18

[7] Karana, E. and P. Hekkert, User-material-product interrelationships in attributing meanings. International Journal of Design, 2010. 4(3).

[8] van Kesteren, I., P.J. Stappers, and S. de Bruijn, Materials in products selection: tools for including user-interaction in materials selection. International Journal of Design, 2007. 1(3).

[9] Ashby, M. and K. Johnson, The Art of Materials Seletion, MaterialsToday. Elsevier, 2003. 12. https://doi.org/10.1016/S1369-7021(03)01223-9 